\title{
A BALESTILHA DESCRITA NA OBRA CHRONOGRAPHIA REPERTORIO \\ DOS TEMPOS (1603): DISCUSSÕES INICIAIS SOBRE O SABER INCORPORADO NO INSTRUMENTO
}

\section{THE CROSS-STAFF DESCRIBED IN THE OEUVRE CHRONOGRAPHIA REPERTORIO DOS TEMPOS (1603): INITIAL DISCUSSIONS ON KNOWING INCORPORATED IN THE INSTRUMENT}

\author{
Ana Carolina Costa Pereira ${ }^{1}$ \\ Universidade Estadual do Ceará \\ Antonia Naiara de Sousa Batista ${ }^{2}$ \\ Instituto Federal de Educação, Ciência e Tecnologia do Ceará \\ Isabelle Coelho da Silva ${ }^{3}$ \\ Instituto Federal de Educação, Ciência e Tecnologia do Ceará
}

\section{Resumo}

Uma das tendências disseminadas e discutidas na busca por uma melhora na educação é a história da matemática. Contudo, para utiliza-la é necessário prudência, pois o educador precisa ter definido o tipo de historiografia que irá adotar. A partir de uma perspectiva historiográfica atualizada, podemos pensar na produção de interfaces entre história da matemática e ensino, em que a construção do conhecimento se dará em torno do objeto de estudo. Para isso, vislumbramos nos instrumentos matemáticos, em particular na balestilha, uma possibilidade de atrelar a teoria e prática dos conceitos matemáticos estudados em sala de aula. Portanto, esse artigo objetiva apresentar a balestilha descrita na obra Chronographia repertorio dos tempos, escrita em 1603 pelo matemático e cosmógrafo português Manoel de Figueiredo. Assim, propomos discutir o saber e o fazer incorporado neste instrumento, buscando articular conceitos matemáticos em sala de aula por meio de interfaces. Dessa forma, esperamos provocar uma reflexão sobre a importância da prática no ensino, mostrando uma possibilidade de mobilizar conhecimentos através de instrumentos náuticos, no nosso caso, a balestilha.

Palavras-chaves: Chronographia repertorio dos tempos. Balestilha. História da Matemática, Ensino da Matemática.

\section{Abstract

\footnotetext{
${ }^{1}$ E-mail: carolina.pereira@uece.br

${ }^{2}$ E-mail: antonianaiarabatista@yahoo.com.br

${ }^{3}$ E-mail: isabellecoelhods@gmail.com
}

One of the trends disseminated and discussed in the search for an improvement in education is the history of mathematics. However, to use it, prudence is necessary, since the educator must have defined the type of historiography he will adopt. From an 
updated historiographic perspective, we can think about the production of interfaces between the history of mathematics and teaching, in which the construction of the knowledge will take place around the object of study. For this, we envisage in the mathematical instruments, in particular in the Cross-staff, a possibility of linking the theory and the practice of the mathematical concepts studied in the classroom. Therefore, this article aims to present the Cross-staff described in the oeuvre Chronographia repertorio dos tempos..., written in 1603 by the Portuguese cosmographer Manoel de Figueiredo. Thus, we propose to discuss the knowing and the doing incorporated in this instrument, seeking to articulate mathematical concepts in the classroom through interfaces. In this way, we hope to provoke a reflection on the importance of practice in teaching, showing a possibility of mobilizing knowledge through nautical instruments, in our case, the Cross-staff.

Keywords: Chronographia repertorio dos tempos. Cross-staff. History of Mathematics, Mathematics teaching.

\title{
Introdução
}

No cenário da Educação Matemática, diversas tendências teóricas nasceram de sua solidificação como um campo de investigação, sendo elas, a Modelagem Matemática, a Etnomatemática, a História da Matemática, Didática da Matemática, entre outras (PAIS, 2002). Em se tratando da história da matemática, é perceptível o aumento de trabalhos desenvolvidos nessa área que buscam disseminar não só aspectos históricos dos conteúdos, mas articular história e ensino, de maneira prática e palpável.

A História da Matemática é apontada como uma importante ferramenta para atividades em sala de aula. Entretanto, devemos ter prudência quando desenvolvemos trabalhos sobre aspectos históricos da matemática voltados para a área da Educação Matemática, pois segundo Baroni e Nobre (1999, p.132),

\begin{abstract}
Ao desenvolvermos estudos relativos às contribuições da História da Matemática para a Educação Matemática, percebemos que é necessário muita cautela, pois pode-se incorrer no erro de simplesmente assumir a História da Matemática como elemento motivador ao desenvolvimento do conteúdo. Sua amplitude extrapola o campo da motivação e engloba elementos cujas naturezas estão voltadas a uma interligação entre o conteúdo e sua atividade educacional.
\end{abstract}

Assim, o desenvolvimento da história da matemática tende a agregar elementos que vão além do entusiasmo e da admiração sobre certos conceitos matemáticos, promovendo justificativas e "por quês" concretos que solidificam essa relação entre ensino e conteúdo. Seu emprego de forma apropriada por meio de fontes seguras e atuais, pode proporcionar entre os alunos, um olhar mais crítico em relação à matemática propriamente dita e à construção do seu conhecimento. 
Nesse sentido, a articulação entre história da matemática e ensino pode oferecer potencialidades didáticas que poderão refletir diretamente na maneira de idealizar certos conceitos matemáticos diminuindo determinados problemas deparados na sua evolução. Nessa relação, precisamos ter definido qual vertente historiográfica, tradicional ou atualizada, o qual o pesquisador ou docente irá se basear para desenvolver essa efetiva articulação.

A história da matemática sobre uma vertente tradicional tende a vislumbrar o passado com o conhecimento matemático que temos hoje, sem realizar nenhuma contextualização no período histórico em que o objeto de estudo estava inserido. Enquanto que, a vertente atualizada procurar contextualizar no passado o objeto de pesquisa, na busca de identificar os conhecimentos matemáticos que tiveram influência sobre o mesmo, dando importância ainda, aos aspectos políticos, sócias, econômicos, culturais, religiosos, entre outros, que contribuíram para o desenvolvimento do assunto trabalhado (SAITO, 2015).

Fazendo o uso da perspectiva historiográfica atualizada podemos construir o conhecimento matemático em articulação com o cenário histórico da Matemática, permitindo analisar a mesma em sua "prática filosófica, científica e social, fornecendo ao discente a compreensão do papel que ele desempenha no mundo". (PEREIRA, 2016, p. 2).

Assim, é por meio da perspectiva atualizada que podemos vislumbrar a construção de interfaces para articular história da matemática e ensino. E essa construção de interface pode ser gerada em torno do objeto de estudo, que no nosso caso, iremos tratar acerca de instrumento náutico conhecido por balestilha, objetivando conhecer o saber-fazer presente no instrumento.

Assim, é por meio da perspectiva atualizada que podemos vislumbrar a construção de interfaces para articular história da matemática e ensino. E essa interface nos possibilita conhecer o saber matemático e o fazer prático do período no qual objeto de estudo estava sendo contextualizado. Nesta pesquisa, o nosso estudo se dará em torno de um instrumento náutico, conhecido por balestilha, de uso entre os séculos XVI e XVIII.

A mesma aparece citada em diversas obras, inclusive sua graduação, dentre elas, podemos destacar: Tratados como o In Hoc Opere Haec Continentur: Nova Translatio 
Primi Libri Geographicae Cl Ptolomaei de Johann Werner, escrito em 1514, e publicado em 1522; Chronographia repertorio dos tempos, Manoel de Figueiredo, escrito em 1603 e publicado em 1605.

Dessa forma, esse artigo tem o intuito apresentar a balestilha descrita na obra Chronographia repertorio dos tempos, do cosmógrafo português Manoel de Figueiredo, escrita em 1603. Nela, é descrita a graduação e construção da balestilha sob o olhar da matemática do início do século XVII, final da Era das grandes navegações. Propõe-se ainda fazer uma discussão inicial acerca do saber e do fazer incorporado no instrumento, na busca por interfaces que permitam a articulação dos conceitos matemáticos através deste em sala de aula.

\section{Instrumentos matemáticos e sua relação com a história e o ensino}

Estudos envolvendo instrumentos históricos no Brasil, em particular do campo da matemática, ainda está em expansão. Segundo Pereira (2016) percebe-se que houve um aumento visível de trabalhos abrangendo a temática a partir de 2010, em periódicos e anais de eventos específicos da área da matemática.

Se adentrarmos para os fundamentos etimológicos da palavra instrumento, em uma definição ampla está relacionada "a um aparato ou aparelho com que se realiza algum trabalho ou se faz algumas observações e medições. Entretanto, ao longo da história, os instrumentos enfrentaram questões de credibilidade, de exatidão, de terminologia, de função, de preservação, entre outros aspectos" (PEREIRA, MARTINS, 2017, p. 28). Entretanto, Saito (2016, p. 4) traz uma definição funcional para o instrumento, em particular o científico “(...) uma ferramenta ou aparato utilizados em laboratório para realizar observações e experimentos; ou ainda, como uma ferramenta que nos permite medir comprimento, peso, e outros fenômenos naturais, tais como pressão, temperatura, força etc".

A nomenclatura "instrumentos científicos" foi inicialmente utilizada durantes dos séculos XIX e XX em decorrência das necessidades profissionais (fabricantes, comerciantes e distribuidores, curadores de museus, entre outros) envolvendo questões econômicas. A organização desses instrumentos em museus e exposições exerceu uma função importante para essa definição nessa época. Segundo Van Helden (1983, p. 49):

A palavra instrumento tem uma variedade de significados, todos agrupados em torno da noção de ferramenta ou dispositivo. Quando nós modificamo-os 
para instrumentos científicos, queremos dizer um dispositivo usado por cientistas para investigar a natureza qualitativa ou quantitativamente. Nós tacitamente supomos que: (1) há um apropriado, até mesmo essencial, lugar para tais dispositivos no estudo da natureza visto que os sentidos humanos sozinhos são demasiado limitados para a maioria das investigações científicas; (2) os resultados ou leituras obtidas com eles estão geralmente fora de questão; (3) instrumentos científicos são baseados em princípios científicos indiscutíveis, como a lei da alavanca; (4) instrumentos mais recentes são mais precisos, poderosos, ou convenientes que os mais velhos, o fator limitante geralmente sendo o estado da arte em tecnologia contemporânea.

Diferentemente como pensamos hoje, o termo "instrumento científico" não está relacionado com um artefato utilizado por um cientista. A partir da invenção do microscópio e telescópio; do barômetro, da bomba de ar e do relógio de pendulo, nos primeiros anos do século XVII, os instrumentos tiveram um aumento considerável, quanto a sua importância na sociedade (WARNER, 1990; VAN HELDEN, 1983).

Foi no decorrer dos séculos XVI e XVII que os artefatos destinados "instrumento científico" foram classificados nas categorias: instrumentos matemáticos, óticos e filosóficos. No que se refere ao termo instrumento matemático, Taub (2009) direciona a aparatos como bússolas e relógios de sol. Essa terminologia foi utilizada até o final do século XIX na qual foi substituído pela expressão "instrumento de engenharia". Os instrumentos óticos referem-se a lentes espelhos, primas, etc. E os instrumentos filosóficos são objetos utilizados na filosofia experimental.

Pereira e Martins (2017, p. 30), fazem uma classificação especifica dos "instrumentos científicos" denominados matemáticos que eram nos séculos XVI e $\mathrm{XVII}^{4}$ ditos como:

(...) aparatos de desenho (compassos, esquadros, réguas, transferidores, outros), cálculo, relógio de sol e utilizados para a astronomia (octante, sextante, esfera Armilar, outros), agrimensura (dioptra; galvanômetro, goniográfico, teodolito, outros), navegação (astrolábios, quadrantes, corda da índia ou Kamal, balestilha, outros), guerra, arquitetura, entre outros.

Nesse período além de apareceram novos métodos matemáticos e experimentais, as grandes áreas das ciências, tais como, Matemática, Química, Física, Biologia, entre outros, foram redefinidas, ocasionando o aparecimento dos instrumentos no cenário

\footnotetext{
${ }^{4}$ Os instrumentos matemáticos eram concebidos nessa época como um "corpo de conhecimento matemático de modo que as matemáticas eram muitas" (SAITO, 2016, p, 5). Dessa forma, muitos desses instrumentos estavam ligados a astronomia e navegação, por esse motivo alguns eram utilizados em ambas as áreas.
} 
científico, facilitando a resolução de problemas matemáticos, observacionais e experimentais (SAITO, 2016).

Em relação aos instrumentos matemáticos relacionados as tarefas do dia-a-dia, Bennett (2003) ressaltam que eles eram utilizados por profissionais, tais como agrimensores, engenheiros militares, navegadores, astrônomos, entre outros. Entretanto, no século XVII não era atribuído os tipos de instrumentos científicos em termos de saber, relacionado ao papel da descoberta, diferença entre o saber e o fazer dos instrumentos matemáticos pode ser o início de uma caracterização adequada da matemática prática.

Dessa forma, instrumentos matemáticos estudados no seu cerne envolvendo o saber e o saber pode adentar a educação, os mesmos proporcionam um importante papel na interface entre a história e ensino. Seu estudo possibilita a articulação entre a teoria, a prática e a experimentação levando o discente a produzir o saber não da construção, mas também elementos que conduz e difunde diferentes formas de conhecimento.

Nesse sentido, o instrumento matemático, envolvendo o conhecimento teórico e prático, permite que questões epistemológicas, axiológicas e antológicas sejam estudadas dentro de uma interface entre história e ensino de matemática.

\section{Conhecendo a balestilha}

A balestilha teve sua nomenclatura variada dependendo da dimensão na qual estava sendo empregada, desde baculus geometricus ou baculus Jacob, para medições realizadas na geodesia, e radius astronomicus, quando aplicada na astronomia. Seu termo primitivo balhestilha, se originou do termo português balhesta, que por sua vez decorreu do latim balista. (NUNES, 2005).

Segundo Bruyns e Dunn (2009), dentre os séculos XV e XVI, a balestilha aparece na sequência de instrumentos científicos desenvolvidos nesse período como sendo a terceira, vindo posteriormente, ao quadrante náutico e ao astrolábio, que obtiveram menções ainda no século XV. O autor ressalta ainda que a mesma teria sido inserida nas navegações em meados de 1515.

Não se sabe ao certo a data do surgimento desse instrumento, mas foi a partir, de uma obra escrita por Johannes Werner (1468 - 1522), In hoc opere haec continentur: Nova Translatio Primi Libri Geographicae Cl Ptolomaei, escrita em 1514 e publicada 
em 1522, que explicava, "cuidadosamente o instrumento, introduzindo algumas inovações e explicando o tipo de observações em que era útil, em particular na determinação da longitude pelo método das distâncias lunares” (NUNES, 2005, p. 372), que possivelmente tenham colaborado para aparição em Portugal das primeiras balestilhas (Figura 1).

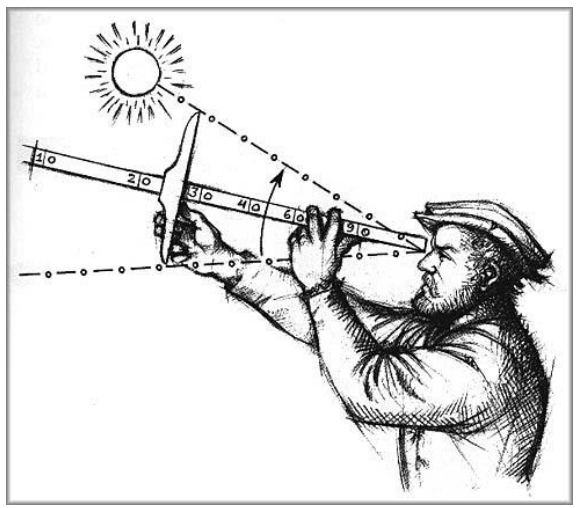

Figura 1 - Medindo a distância do astro em relação a linha do horizonte Fonte: Bruyns (1997, p. s/p)

A finalidade da Balestilha pode ser identificada na fala de Silva (1945, p. 279), "D. João de Castro recomenda a balestilha para tomar a altura do Norte ou do Cruzeiro do Sul. Pedro Nunes acha-a sobretudo boa para medir distâncias entre os astros (Figura 1), menores que 90 graus". Assim, podemos ver que o instrumento dispunha de duas maneiras distintas para observações astronômicas, sendo a primeira realizada tomando por base a linha do horizonte e a segunda colocando as extremidades da soalha nos astros, para obtenção da distância.

A Balestilha era formada por uma vara de madeira com secção quadrada, de aproximadamente $800 \mathrm{~mm}$ de extensão e $16 \mathrm{~mm}$ de largura, juntamente com uma ou mais soalhas ${ }^{5}$, que seriam menores que o virote, com cerca de $40 \mathrm{~mm}$ de largura e com um orifício no seu centro para a inserção da vara (Figura 2). (BRUYNS, DUNN, 2009).

\footnotetext{
${ }^{5}$ No que se faz menção as soalhas da balestilha, ainda é incerto a verdadeira quantidade das mesmas, devido as distintas informações encontradas em diferentes fontes históricas.
} 


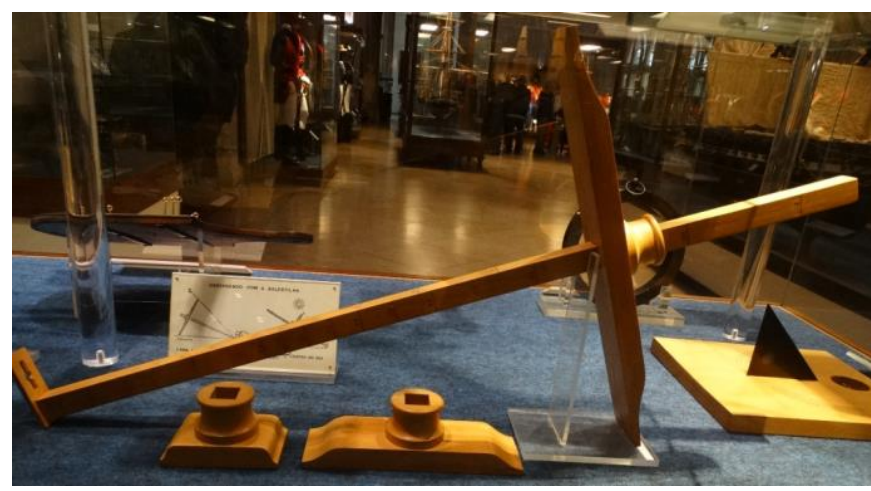

Figura 2 - Vara e soalhas

Fonte: Museu da marinha em Lisboa

Cada face da vara possui uma graduação correspondente a uma soalha especifica, por isso apenas se usava uma soalha por vez. Sua graduação era realizada de 0 a 90 graus. Mesmo com as dificuldades encontradas na hora de centrar o olho na extremidade do virote ou apontar a parte superior da soalha no centro do astro, a Balestilha permaneceu em uso em alto-mar até o final do século XVIII (BRUYNS, DUNN, 2009).

\section{Descrição e graduação da balestilha por Figueiredo (1603)}

A obra Chronographia repertorio dos tempos, do português Manoel de Figueiredo, escrita em 1603 e impressa em 1608, ainda reeditada em 1609, 1614, 1625 e 1632, está dividida em 6 partes com 284 folhas $^{6}$. A primeira parte trata acerca das horas, dias e meses de um ano, a segunda parte traz tópicos relacionados à astronomia, a terceira aborda sobre assuntos da geografia, a quarta trata sobre astrologia, na quinta parte discorre sobre calendários, e por fim, a sexta parte apresenta a graduação da balestilha de maneira geométrica e sua aplicação para medição da distância entre dois astros.

A sexta parte ou o livro sexto contém 12 capítulos num total de 18 folhas (34 página). Figueiredo (1603, p. 266) descreve no título do livro como: "Da fabrica, e uso da balhestilha, ou radio astronômico, e do uso e fabrica, do quadrante geométrico e da fabrica, e uso dos relogios horizontais, verticais, laterais, equinociais, polarea declinantes a todas as partes do mundo, e enclinantes", em que os dois primeiros

\footnotetext{
${ }^{6}$ Ressaltamos que atualmente a obra iria conter 568 páginas.
} 
capítulos versam sobre: da fabrica da balhestilha, ou radio astronômico; e do uso do radio astronômico.

Para a graduação da balestilha, Figueiredo (1603) apresenta uma descrição da marcação do virote partindo de um semicírculo ou um quarto de um círculo. Segundo o autor ${ }^{7}$ :

O radio astronômico, ou balhestilha se fabrica em um semicírculo, ou no quarto de círculo pela seguinte ordem: faça um quarto de círculo em uma taboa $\mathrm{A}, \mathrm{B}$ e $\mathrm{C}^{8}$. Partiremos o arco $\mathrm{BC}$ ao meio, no ponto $\mathrm{D}$. $\mathrm{E}$ do ponto $\mathrm{D}$ até o ponto $\mathrm{B}$ partiremos em quarenta cinco partes iguais. Partiremos primeiro o espaço DB em três partes iguais, e depois cada uma em outras três, e assim ficará partida em nove espaços, e logo cada um deles em cinco partes, e ficará partido em 45 partes iguais. E cada parte delas partiremos ao meio, e serão noventa partes.

Temos que buscar uma taboa muito plana, e lisa, de cedro ou pereiro, em que tracemos a presente demonstração. $\mathrm{E}$ depois de traçada, veremos de que tamanho quero que seja, o pedacinho, que os marinheiros chamam de soalha, e suponho que seja do tamanho da linha GE, cujo meio será o ponto A. Do ponto $\mathrm{E}$ lançaremos uma linha paralela à linha $\mathrm{AB}$, a linha $\mathrm{E}$. $\mathrm{E}$ pondo uma régua muito bem direita, e examinada no ponto $\mathrm{A}$. Em cada parte da quarta BA faremos divisões onde cortara régua na linha $\mathrm{E}$, a qual a linha ficara partida em outras noventa partes como está partido o arco BD: o que teremos muito bem feito como está dito. E pelo conseguinte faremos uma régua quadrada de pau preto, ou de brasil, ou de cedro, a qual tenha todos os quatros lados iguais, e em um dos lados lhe lançaremos três ou quatro linhas paralelas ao comprido. Duas mais chegadas, e outras duas mais apartadas, como mostra a figura HY. E com um compasso the passaremos todos os espaços da linha E. E assim teremos dividida toda a régua em tantas partes em quantas estiver a linha $\mathrm{E}$, as quais partes chamamos graus, e os poremos com suas divisões de dez em dez graus com seus números, como demonstra a presente figura.

O pedacinho, ou soalha será três vezes quanto for a régua, a qual se fará de uma polegada de largo, e a soalha de três, e será de tamanho da linha GE. E no meio lhe faremos um buraco quadrado quanto caiba a régua o mais justo que puderem ser, e assim ficara feito o radio astronômico, ou balhestilha.

\footnotetext{
${ }^{7}$ Apresentamos uma adaptação do capítulo: da fabrica da balhestilha, ou radio astronômico. A linguagem apresentada na obra é um português do início século XVII, entretanto adequamos para uma fala mais contemporânea, visando a melhor compreensão. No restante, foram mantidas as nomenclaturas originais, preservando o pensamento inicial do autor.

${ }^{8}$ Mantivemos a figura e as letras usadas por Figueiredo na versão indicada, entretanto, utilizamos letras maiúsculas para indicar pontos na construção, conforme a figura apresentada, já que no texto o autor utiliza-se de letra minúsculas. A pontuação também foi alterada, pois Figueiredo não utiliza ponto final para finalizar uma ideia.
} 


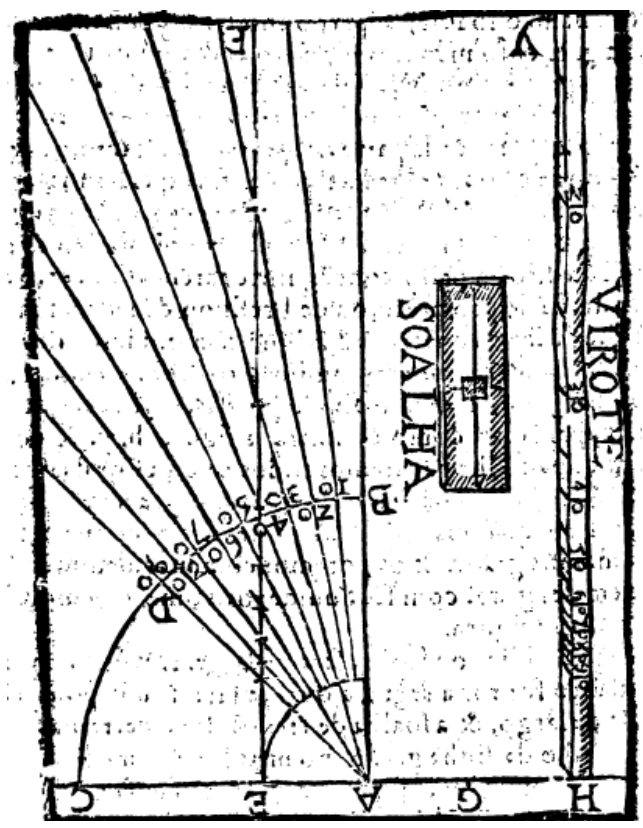

(FIGUEIREDO, 1603, f. 266-267)

Na descrição feita por Figueiredo (1603) além dele apresentar a graduação da balestilha, ele indica o melhor material para sua confecção e a maneira de construí-la. Entretanto, o saber constituído por traz da sua construção não é detalhado por ele. Por exemplo, “(...) partiremos o arco $\mathrm{BC}$ ao meio, no ponto D. E do ponto D até o ponto B o partiremos em quarenta cinco partes iguais" (FIGUEIREDO, 1603, f. 266), não está claro qual material ou procedimento o leitor irá fazer para traçar o ponto médio de $\mathrm{BC}$, ou mesmo para dividir o ângulo em 45 partes iguais.

De fato, a obra de Figueiredo (1603) está caracterizada como um almanaque ${ }^{9}$ que contém informações gerais voltadas para a cosmografia. Ele não é um manual de "faça você mesmo" e nem carrega características dos tratados sobre instrumentos século XVI, sobre fazer e saber, em que o fazer prenominavam nas apresentações dos manuais de instruções. Segundo Saito e Dias (2011, p. 56) os instrumentos no século XVI e XVII

(...) são mais do que simples artefatos. Eles incorporam conhecimentos que revelam a articulação entre o saber e o fazer e, desse modo, sintetizam a produção de conhecimento de uma época. Conhecimento este que pode receber diferentes interpretações e, consequentemente, significados.

\footnotetext{
${ }^{9}$ Os almanaques eram pequenas publicações impressas em largas tiragens que tinham uma periodicidade anual e que eram colocados à venda em locais próprios do livreiro ou eram vendidos pelos vendedores ambulantes (SZESZ, 2008, p. 4),
} 
Essas interpretações dependerão do objetivo e formação do pesquisador envolvido, já que os instrumentos podem ser vistos "como construtores de conhecimento e não apenas como objetos mediadores entre teoria e prática (...)" (SAITO, 2013, p. 1153), pois, exercem diferentes funções na articulação entre esses dois entes.

Assim, é por meio da manipulação do instrumento que se pode identificar diversos conhecimentos matemáticos que estão implícitos não só na parte física, mas também na maneira como e efetuada a sua aplicação. Como consequência, temos a oportunidade de conhecer diferentes aspectos históricos do tipo, social, cultural, religioso, embebidos no âmbito da aplicação desses instrumentos. Desta forma, quando se trabalha com um instrumento e considera o mesmo além da sua materialidade podemos descobrir aspectos históricos e matemáticos que nos permitirão articular o saber e fazer inserido na manipulação do mesmo.

Dessa forma, é evidente que o saber e o fazer inserido na obra de Figueiredo (1603) precisa ser visto e revisto para que seja passível de uso na sala de aula. Apesar de não ser a finalidade do livro, os conhecimentos incorporados na graduação da balestilha, podem ser transformados em passos para a construção geométrica do virote e das soalhas. Um estudo paralelo da fonte histórica incluindo métodos de desenho geométrico usados no século XVI pode ser uma forma de articulação entre história e ensino, diversificando a metodologia utilizada atualmente nas aulas de matemática.

\section{Ponderações finais}

O resgate histórico de determinado conteúdo, método ou mesmo instrumento matemático desenvolvido por uma civilização e disseminado ainda nos dias de hoje, pode nos trazer benefícios educacionais se utilizado de forma correta. $\mathrm{O}$ uso da história da matemática no ensino pode proporcionar diversos benefícios, tais como uma contextualização do conteúdo teórico estudado em sala, um retorno ao passado, uma visualização prática dos conceitos ensinados, entre outros. Assim, a geometria é um exemplo de área da matemática em que percebemos que possível mobilizar conhecimentos através da inserção de aspectos históricos em sala de aula.

As construções geométricas, com o manejo da régua e do compasso, existem a mais de 2000 anos, e sua utilização perpassa épocas e ainda é encontrada atualmente 
para justificar e/ou demonstrar teoremas da geometria. Além de desenvolver a habilidade motora que, aliada à utilização de conceitos de Geometria Plana, propicia a manipulação e a experimentação, as construções geométricas oferecem o aperfeiçoamento do pensamento geométrico.

Nesse estudo, buscamos apresentar a balestilha descrita na obra Chronographia repertorio dos tempos, escrita em 1603 pelo cosmógrafo português Manoel de Figueiredo. Para isso, foi necessário investigar as potencialidades dos instrumentos matemáticos para o ensino, evidenciando o estudo das construções geométricas. Assim, acreditamos que esse artefato pode proporcionar diversas possibilidades de articular história da matemática e ensino, em que essa reflexão pode ser iniciada a partir do texto de Manoel de Figueiredo.

Dessa forma, a obra Chronographia repertorio dos tempos tem potencialidades no que se refere a possível articulação entre a matemática incorporada no instrumento, a história e o ensino. Embora ela não seja um tratado especifico de geometria prática ou instrumento do século XVI e XVII, ela traz a descrição, graduação e construção da balestilha com o olhar da época.

Seu uso didático envolvendo conceitos de geometria por meio das construções precisa ser articulado entre o passado e o presente, na busca pelo conhecimento do contexto no qual o mesmo estava inserido, realizando assim, o movimento do pensamento na construção do conhecimento matemático. A construção dessa interface é o próximo encaminhamento que precisa ser dado para a inserção da balestilha na sala de aula.

\section{REFERÊNCIAS}

BARONI, Rosa Lúcia Sverzut; NOBRE, Sergio Roberto. A Pesquisa em História da Matemática e suas Relações com a Educação Matemática. In: BICUDO, M. A. V. (Org.). Pesquisa em Educação Matemática: concepções \& perspectivas. São Paulo: Editora UNESP, 1999. p. 129-136.

BENNETT, Jim. (2003). Knowing and doing in the sixteenth century: What were instruments for? British Journal for the History of Science, 36, 129-150.

BRUYNS, Willem Fredrik Jacob Mörzer. The Cross-staff: History and Development of a Navigational Instrument. Zutphen: Walburg Pers, 1994. 
Ana Carolina Costa Pereira, Antonia Naiara de Sousa Batista, Isabelle Coelho da Silva

A balestilha descrita na obra Chronographia repertorio dos tempos (1603): discussões iniciais sobre o saber incorporado no instrumento

BRUYNS, Willem Fredrik Jacob Mörzer; DUNN, Richard. Sextants at Greenwich: A Catalogue of the Mariner's Quadrants, Mariner's Astrolabes Cross-staffs, Backstaffs, Octants, Sextants, Quintants, Reflecting Circles and Artificial Horizons in the National Maritime Museum, Greenwich. Oxford: Oxford University Press, 2009.

FIGUEIREDO, Manoel. Chronographia repertorio dos tempos. Portugal, 1603. 296 p.

NUNES, Pedro. Obras, vol. III, (Erratis Orontii Finaei Regii Mathematicarvm Lvtetiae Professoris). Lisboa: Academia das Ciências de Lisboa / Fundação Calouste Gulbenkian, 2005.

PAIS, Luiz Carlos. Didática da Matemática: Uma análise da influência francesa. 2. ed. Belo Horizonte: Editora Autêntica, 2002b. 128 p.

PEREIRA, Ana Carolina Costa. Investigando as potencialidades didáticas dos instrumentos históricos no ensino de Matemática. In: SEMINÁRIO NACIONAL DE HISTÓRIA DA CIÊNCIA E DA TECNOLOGIA, 15., 2016, Florianópolis. Anais... . Florianópolis: Universidade Federal de Santa Catarina, 2016. p. 1 - 16.

PEREIRA, Ana Carolina Costa; MARTINS, Eugeniano Brito. O ensino de aritmética por meio de instrumentos: Uma Abordagem utilizando do Rabdologiae seu numerationis per virgula. São Paulo: Editora da Física, 2017.

SAITO, Fumikazu. História e Ensino de Matemática: Construindo Interfaces. In: SALAZAR, Jesús Flores; GUERRA, Francisco Ugarte. Investigaciones en Educación Matemática. Lima: PUCP, 2016. p. 253-291.

SZESZ, Christiane Marques. Os almanaques populares: leituras e apropriações em Ariano Suassuna. In: ENCONTRO ESTADUAL DE HISTÓRIA, 9., 2008, Porto Alegre. Anais... . Porto Alegre: ANPUH-RS, 2008. p. 1 - 9.

SILVA, Luciano Pereira da. Obras Completas. Lisboa: Tipografia dos Caminhos de Ferro e Estado, 1923. (Volume II).

SAITO, Fumikazu. Instrumentos e o 'saber-fazer' matemático no século XVI, in: Ciência, Tecnologia e Cultura: Outro desenvolvimento é possível? Anais do V Simpósio Nacional de Tecnologia e Sociedade. Curitiba, UTFPR/ESOCITE.BR, 2013, pp. 1151-1160.

SAITO, Fumikazu. História da matemática e suas (re)construções contextuais. São Paulo: Editora Livraria da Física, 2015. 259 p.

SAITO, Fumikazu; DIAS, Marisa da Silva. Articulação de entes matemáticos na construção e utilização de instrumento de medida do século XVI. Natal: Sociedade Brasileira de História da Matemática, 2011. 
TAUB, Liba. On scientific instruments. Studies in History and Philosophy of Science. 40 (2009) 337-343.

VAN HELDEN, Albert. The birth of the modern scientific instrument, 1550-1700. In J. G. Burke (Ed.), The uses of science in the Age of Newton. Berkeley: University of California Press, p. 49-84), 1983.

WARNER, Deborah Jean. What is a scientific instrument, when did it become one, and why?. British Journal for the History of Science, v. 23, London, p. 83-93, 1990.

WERNER, Johann. In hoc opere haec continentur: Nova Translatio Primi Libri Geographicae $\mathrm{Cl}$ Ptolomaei. Nurembergae: Friedericum Peypus, Lucar Alantfee Ciuis \& Bibliopolae Vienneñ, 1522. 